compared to HLA-B15 patients. Accordingly, the genotype (presence of HLA-B27 or HLA-B15) and phenotype (axial or peripheral involvement) may help physicians when considering a targeted therapy of SpA patients with IL-17 inhibition in a context of personalized medicine

Disclosure of Interest: None declared

DOI: 10.1136/annrheumdis-2017-eular.5628

\section{THU0053 SELECTIVE ACTIVATION OF AN AMPK-CREB-NRF2-DEPENDENT PATHWAY BY CELECOXIB INDUCES VASCULOPROTECTIVE GENES AND MITIGATES AGAINST CARDIOVASCULAR RISK}

F. Alrashed, D. Calay, C.C. Thornton, A. Bauer, A. Kiprianos, D.O. Haskard, A. Seneviratne, J.J. Boyle, J.C. Mason. Rheumatology and Vascular Sciences, Imperial College London, London, United Kingdom

Background: Although concern remains about the athero-thrombotic risk posed by COX-2-selective inhibitors (COXIBs), the recent PRECISION trial demonstrated non-inferiority of moderate dose celecoxib when compared to naproxen and ibuprofen with respect to cardiovascular safety, with fewer actual CV events recorded for celecoxib. Moreover, celecoxib proved significantly safer than either comparator in regard to gastrointestinal events ${ }^{1}$. Given the markedly different cardiovascular risk associated with celecoxib and rofecoxib, we investigated the hypothesis that, in addition to cyclo-oxygenase inhibition, celecoxib specifically activates COX-2-independent AMP kinase (AMPK) signalling to exert protective effects in the vascular endothelium

Objectives: To investigate COX-2-independent vasculoprotective signalling pathways activated by celecoxib in human endothelium.

Methods: In vitro studies of celecoxib, rofecoxib, ibuprofen and naproxen were performed on human umbilical vein and human aortic endothelial cells (HUVEC and HAEC). Inhibition of signalling pathways was achieved using siRNA. The vascular effects of celecoxib in vivo were studied in C57BI/6 mice fed celecoxib (1000 ppm) or control chow (48 hrs). Aortic tissue was snap-frozen and sections studied by immunofluorescence confocal microscopy.

Results: At therapeutically relevant concentrations celecoxib $(1-10 \mu \mathrm{M})$ induced the vasculoprotective protein heme oxygenase-1 (HO-1) in HUVEC and HAEC (EC) $(p<0.01)$. In contrast, rofecoxib and the commonly used non-selective NSAIDs ibuprofen and naproxen failed to induce HO-1. Celecoxib derivative 2,5dimethyl-celecoxib (DMC), which lacks COX-2 inhibition, also upregulated HO-1, implicating a COX-2-independent mechanism. Immunoblotting demonstrated that celecoxib and DMC induce AMPK $\alpha^{(\text {Thr172) }}$ and CREB-1(Ser133) phosphorylation leading to Nrf2 nuclear translocation $(p<0.05)$. These responses were not seen with ibuprofen or naproxen, while siRNA depletion of AMPK $\alpha$ abrogated celecoxib-mediated CREB and Nrf2 activation $(p<0.05)$. Acting via the same pathway, celecoxib induced additional cytoprotective genes including $\mathrm{H}$-ferritin. In vivo, celecoxib similarly increased $\mathrm{HO}-1$ and $\mathrm{H}$-ferritin in murine aortic endothelium when compared to control-fed mice $(p<0.05)$. Functionally, celecoxib treatment

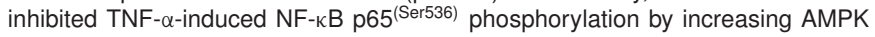
activity. This attenuated VCAM-1 upregulation via induction of $\mathrm{HO}-1$, as revealed by HO-1 siRNA $(p<0.05)$. Similarly, celecoxib prevented the IL-1-mediated increase of IL- 6 mRNA $(p<0.01)$. These responses were not seen with ibuprofen or naproxen.

Conclusions: Celecoxib induces anti-inflammatory, anti-oxidant proteins $\mathrm{HO}-1$ and $\mathrm{H}$-ferritin in human vascular endothelium via a novel AMPK-CREB-Nrf2dependent pathway. This mechanism may contribute to the important and marked differences in cardiovascular risk between celecoxib and rofecoxib. Understanding mechanisms underlying NSAID heterogeneity may ultimately lead to the development of safer anti-inflammatory drugs.

References:

[1] Nissen SE et al. Cardiovascular Safety of Celecoxib, Naproxen, or Ibuprofen for Arthritis. N Engl J Med. 2016;375:2519-29.

Disclosure of Interest: None declared

DOI: 10.1136/annrheumdis-2017-eular.2841

\section{THU0054 UTILITY OF SEROLOGICAL PARAMETERS IN GIANT CELL} ARTERITIS FOR PREDICTING DISEASE COMPLICATIONS

K. Lakota ${ }^{1,2}$, J. Feichtinger ${ }^{3,4}$, B. Burja ${ }^{1}$, T. Kuret ${ }^{1}$, P. Žigon ${ }^{1}$, Ž. Rotar ${ }^{1}$ R. Ješe ${ }^{1}$, S. Sodin-Šemrl ${ }^{1,2}$, S. Čučnik ${ }^{1,5}$, G. Thallinger ${ }^{3,4}$, M. Tomšič ${ }^{1,6}$ A. Hočevar ${ }^{1}$. ${ }^{1}$ Department of Rheumatology, University Medical Centre Ljubljana, Ljubljana; ${ }^{2}$ FAMNIT, University of Primorska, Koper, Slovenia, ${ }^{3}$ Institute of Molecular Biotechnology, Graz, Austria, Graz University of Technology; ${ }^{4}$ OMICS Center Graz, BioTechMed Graz, Graz, Austria; ${ }^{5}$ Faculty of Pharmacy; ${ }^{6}$ Faculty of Medicine, University of Ljubljana, Ljubljana, Slovenia

Background: Giant Cell Arteritis (GCA) is a systemic vasculitis affecting primarily large and medium sized arteries. Immediate high dose steroid treatment may prevent urgent complications, such as vision loss and cerebrovascular insults ${ }^{1-3}$. However, there is a clear lack of data on predicting serological markers and their association to clinical complications.

Objectives: To investigate serological parameters that support clinicians in predicting complications in a large, clinically well-characterized set of untreated GCA patients at time of diagnosis.
Methods: The study included 98 GCA patients (67\% female) with a median (IQR) age 74.1 (67.3-78.8) years and a median (IQR) symptome duration time of 30 (20-90) days). Healthy blood donors (HDs, $n=52,61.5 \%$ female, median (IQR) age of 41.95 (20.4-63.1) years) served as controls. GCA complications studied were visual disturbances (including permanent loss of vision), relapses, peripheral artery involvement and claudication. Levels of 27 serum analytes were measured using Luminex xMAP Technology. Interleukin-6 (IL-6) and serum amyloid A (SAA) levels were tested using ELISA and nephelometry, respectively.

Results: The highest, significantly elevated analytes in GCA vs. HDs were SAA (85-fold > HDs mean values), IL-23 (63-fold) and IL-6 (11-fold). IL-13, $\alpha$-fetoprotein and MMP-2 were significantly decreased in GCA, while levels of IL-2, IL-17A and TNF- $\alpha$ were unchanged. PCA analysis revealed a signature analyte profile positioning towards the HD cluster. SAA, CRP, haptoglobin, ESR, thrombocyte \# and matrix metalloproteinase-1 (MMP-1) all negatively associated with visual disturbances, confirming our previous data. Age showed significant association to permanent visual loss, with older patients being more affected ${ }^{3}$. SAA, CRP and ESR at presentation were found to be predictive of relapsing disease, while MMP-2 negatively associated with relapse. VCAM-1, $\alpha$-fetoprotein, MARCO and IL-27 were all negatively associated with peripheral artery involvement. MMP-2 and MARCO showed positive association with claudication, while IL-18 was negatively associated.

Conclusions: In our large study of untreated GCA patients we highlight the importance of using serological acute phase parameters, MMPs and other analytes for predicting complications.

References:

[1] Weyand CM, Goronzy JJ. Medium- and large-vessel vasculitis. N Engl J Med 2003, 349:160-169.

[2] Nesher G: The diagnosis and classification of giant cell arteritis. J Autoimmun 2014, 48-49:73-75.

[3] Hocevar A et al. Do Early Diagnosis and Glucocorticoid Treatment Decrease the Risk of Permanent Visual Loss and Early Relapses in Giant Cell Arteritis: A Prospective Longitudinal Study. Medicine (Baltimore) 2016, 95:e3210.

Disclosure of Interest: None declared DOI: 10.1136/annrheumdis-2017-eular.3467

\section{THU0055 LOW MOLECULAR WEIGHT BAFF SIGNALING INHIBITORS AMELIORATE IL-6, IL-10 AND IGG PRODUCTION IN VITRO AND IN VIVO MODELS OF AUTOIMMUNE DISEASES}

K. Yoshimoto ${ }^{1}$, K. Suzuki ${ }^{1}$, K. Sugahara ${ }^{2}$, T. Takeuchi ${ }^{1}$. ${ }^{1}$ Keio University School of Medicine, Tokyo; ${ }^{2}$ Mitsubishi Tanabe Pharma Corporation, Yokohama, Japan

Background: In our previous study, we reported that soluble BAFF (sBAFF) robustly enhanced IL-6 production by peripheral monocytes of patients with primary Sjogren's syndrome (pSS) and that the expression level of a BAFF receptor (BR3) was significantly elevated in pSS monocytes. We also found that the proportion of BR3-positive monocytes to total monocytes was positively and significantly correlated with the serum lgG level of pSS patients. Investigation of the interaction of monocytes and $B$ cells showed that IgG production by $B$ cells was enhanced by sBAFF-stimulated monocytes. These data collectively suggest that the elevated expression of BR3 on monocytes is involved in IgG overproduction by $B$ cells which is often observed in pSS, and that BAFF signaling via BR3 is a possible therapeutic target to treat pSS. A high throughput screening of a low molecular weight compound library successfully discovered two pyrrolopryimidine derivatives, BIK12 and BIK13, which inhibit SBAFF binding to $B R 3$. We found that these compounds inhibited not only IL-6 production by BAFF-stimulated monocytes, but also IgG production by $B$ cells co-cultured with the monocytes.

Objectives: To elucidate the mechanism of inhibitory activities of these compounds on BAFF signaling pathways, we measured production of IL-10 as well as IL-6 by monocytes in vitro, both of which work for B cell activation. In addition, we analyzed in vivo effects of the compounds on production of autoantibody and cytokines in autoimmune disease model mice.

Methods: Peripheral monocytes and B cells were prepared from healthy individuals. The monocytes were stimulated with SBAFF and cultured in vitro with or without peripheral $B$ cells in the presence of BIK-12 or BIK-13. The amounts of IL-6, IL-10 and IgG were measured by ELISA. BIK-13 was administered intraperitoneally to MRL/lpr mice, an animal model of autoimmune diseases, three times a week for 6 months. Serum levels of an anti-ds DNA antibody, IL-6 and IL-10 were measured by ELISA.

Results: sBAFF enhanced the production of not only IL-6, but also IL-10 by peripheral monocytes in vitro. As expected, BIK12 and BIK-13 significantly suppressed production of IL- 6 and IL-10 by BAFF-stimulated monocytes in vitro in a dose dependent manner. Notably, IgG production by $\mathrm{B}$ cells co-cultured in vitro with sBAFF-stimulated monocytes was significantly suppressed by these compounds. The compounds did not exhibit toxicities to the cells in the dose range. These data suggest that BAFF signaling via BR3 lead to production of IL-6 and IL-10 in monocytes, and the cytokines in turn mediates the signal to B cells resulting in IgG overproduction. Interestingly, serum levels of an anti-dsDNA antibody, IL-6 and IL-10 in MRL/Ipr mice received BIK13 simultaneously declined as compared to control mice. These data collectively suggest that the compound was also efficacious in vivo.

Conclusions: BIK-13, a pyrrolopyrimidine derivative, suppressed IgG production 
by $B$ cells through affecting monocytes. IL- 6 and/or IL-10 may intermediate the effect. Our findings strongly suggest that BAFF signaling via BR3 is a possible therapeutic target for drug discovery to treat pSS or other intractable autoimmune diseases which accompany hypergammaglobulinemia. Moreover, these compounds may provide novel tools to explore the pathological mechanism of the development of these autoimmune diseases.

Disclosure of Interest: K. Yoshimoto: None declared, K. Suzuki: None declared, K. Sugahara Employee of: Mitsubishi Tanabe Pharma Corporation, T. Takeuchi Grant/research support from: Mitsubishi Tanabe Pharma Corporation

DOI: 10.1136/annrheumdis-2017-eular.4176

\section{THU0056 FREE FATTY ACIDS PROMOTE INFLAMMATION VIA OSTEOBLASTS AND OSTEOCLASTS FROM PATIENTS WITH RHEUMATOID ARTHRITIS OR OSTEOARTHRITIS}

K. Frommer ${ }^{1}$, A. Schäffler ${ }^{2}$, U. Lange ${ }^{1}$, S. Rehart ${ }^{3}$, J. Steinmeyer ${ }^{4}$ M. Rickert ${ }^{4}$, U. Müller-Ladner ${ }^{1}$, E. Neumann ${ }^{1} \cdot{ }^{1}$ Dept. of Internal Medicine and Rheumatology, Kerckhoff-Klinik, Justus-Liebig-University of Giessen, Bad Nauheim; ${ }^{2}$ Dept. of Internal Medicine III, Endocrinology, Diabetes, Metabolism, Justus-Liebig-University of Giessen, Giessen; ${ }^{3}$ Dept. of Orthopaedics and Trauma Surgery, Agaplesion Markus Hospital, Frankfurt; ${ }^{4}$ Dept. of Orthopaedics and Orthopaedic Surgery, University Hospital Giessen and Marburg, Giessen, Germany

Background: Various inflammatory cardiovascular and metabolic diseases such as atherosclerosis, coronary heart diseases and type 2 diabetes are associated with chronically elevated free fatty acid (FFA) levels. With inflammation being a factor in pathological bone loss, FFA may also be contributors to bone loss in osteoarthritis (OA) and/or rheumatoid arthritis (RA).

Objectives: To investigate whether FFA have an influence on osteoblasts and osteoclasts from patients with RA or OA, in a way that may alter bone degradation in these diseases.

Methods: Primary osteoblasts $(\mathrm{OB})$ were isolated from cancellous bone of $\mathrm{OA}$ and RA patients undergoing knee joint surgery. Osteoclasts $(\mathrm{OC})$ were differentiated from peripheral blood mononuclear cells (PBMC). $\mathrm{OB}$ and $\mathrm{OC}$ were stimulated with the saturated FFA palmitic acid (PA) and the unsaturated FFA linoleic acid (LA) (100 $\mu \mathrm{M}$ each). Immunoassays were used to quantify protein secretion. mRNA expression levels were quantified by real-time PCR. Mineralization activity was quantified using Alizarin Red S staining, differentiated OC were quantified by counting TRAP-positive multinuclear cells (>2 nuclei). Toll-like receptor (TLR) 4 and TLR2 were blocked by neutralizing antibodies.

Results: When stimulated with FFA, OB from RA and OA patients secreted higher amounts of the proinflammatory cytokine IL-6 (up to 9-fold) and the chemokines IL-8 (up to 221-fold), GRO-a (from below detection level to detectable levels) and MCP-1 (up to 16-fold). Differences in the degree of response were more dependent on the patient than the disease. RANKL as well as OPG, OB-secreted modulators of OC differentiation, as well as $\mathrm{OB}$ differentiation markers (e.g. osterix, osteocalcin) were not influenced by FFA on mRNA or protein level. The effect of FFA on mineralization activity of $\mathrm{OB}$ varied between patients, yet overall there was no significant difference between FFA-treated and untreated $\mathrm{OB}$. Expression of the two Wnt signaling molecules, axin-2 and b-catenin, was not changed by PA or LA, suggesting no involvement of the Wnt signaling pathway in the effects observed by FFA in OB. On the other hand, TLR4 blockade significantly reduced PA-induced IL-8 secretion by OB (by 93\%), while blocking TLR2 had no effect. In both RA and OA OC, IL-8 secretion was significantly enhanced by PA and LA, with a clear time-dependency within the differentiation process for RA OC but not for OA OC. The number of TRAP positive multinuclear cells decreased for RA OC by approx. $50 \%$, which was in agreement with the reduced TRAP secretion by a factor of $2-3$ at d14. mRNA expression of various osteoclast activity markers (CLCN7, CTSK, TCIRG) was not altered.

Conclusions: Inflammation is promoted by FFA via both $\mathrm{OB}$ and $\mathrm{OC}$ from patients with RA or OA, thus possibly indirectly contributing to bone loss while no direct effect on $\mathrm{OB} / \mathrm{OC}$ activity could be observed. In $\mathrm{OB}$, these effects are probably mainly mediated by TLR4, while TLR2 and Wnt pathways do not play a role.

Disclosure of Interest: None declared

DOI: 10.1136/annrheumdis-2017-eular.3488

\section{THU0057 LIPID PROFILING OF PLASMA IN RHEUMATOID ARTHRITIS PATIENTS BY LIQUID CHROMATOGRAPHY-TANDEM MASS SPECTROMETRY}

K. Murakami ${ }^{1}$, I. Murakami ${ }^{1}$, A. loan-Facsinay ${ }^{2}$, M. Giera ${ }^{3}$, M. Hashimoto ${ }^{4}$ A. Yoshida ${ }^{1}$, T. Usui ${ }^{1}$, N. Kuramoto ${ }^{1}$, R. Nakashima ${ }^{1}$, Y. Imura ${ }^{1}$, H. Yoshifuji ${ }^{1}$, K. Ohmura ${ }^{1}$, T. Mimori ${ }^{1} .{ }^{1}$ Department of Rheumatology and Clinical Immunology, Graduate School of Medicine, Kyoto University, Kyoto, Japan; ${ }^{2}$ Department of Rheumatology; ${ }^{3}$ Center for Proteomics and Metabolomics, Leiden University Medical Center, Leiden, Netherlands; ${ }^{4}$ Department of the Control for Rheumatic Diseases, Graduate School of Medicine, Kyoto University, Kyoto, Japan

Background: Previously it has been described that lipid and lipid mediators are present in synovial fluid from patients with rheumatoid arthritis (RA). It is, however, currently unknown to what extent these lipid mediators are involved in disease pathophysiology.

Objectives: The aim of this study is to clarify which lipid mediators in plasma correlate with disease activity of RA.

Methods: We obtained blood from RA patients registered in the KURAMA (Kyoto University Rheumatoid Arthritis Management Alliance) cohort. None of the patients was treated with glucocorticoids or NSAIDs, both of which could affect lipid metabolism. Targeted lipidomics, using a LC-MS/MS (liquid chromatographytandem mass spectrometry) platform was used for the identification of lipids present in the patients' plasma. SDAI (simplified disease activity index) was examined in this cohort and lipidomics profiling and disease status were combined. Data were statistically analyzed by Spearman's rank correlation coefficient test or multivariate regression analysis.

Results: Twenty-six RA patients were enrolled; female ratio: $84 \%$, mean age: 63.0 years old, mean disease duration: 18.7 years and mean SDAI 5.26. In this group, patients age was significantly correlated with SDAI ( $p$ value $=0.005$, Spearman's rho $=0.552$ ). By LC-MS/MS analyses, 23 lipid components were identified and quantified. Multivariative regression analysis (Standard Least Squares) revealed that 19,20-diHDPA (Dihydroxydocosapentaenoic acid) and 14,15-diHETE (Dihydroxyeicosatetraenoic acid) significantly explained SDAI score independently of sex and age.

Among the composite measure for SDAI, the best correlated component with TJC (tender joint count) was LA (Linoleic acid, $p=0.002$, rho $=-0.611$ ), that with patient VAS (visual analogue scale) was 19,20 -diHDPA $(\mathrm{p}=0.032$, rho $=0.440)$, and that with CRP was DHA (Docosahexaenoic acid, $\mathrm{p}=0.021$, rho $=-0.452$ ).

Additionally, principal component analysis was carried out. In the first primary component (PC1), absolute eigenvecotor values of AdA (Adrenic acid), ALA (Alpha-linolenic acid), DHA, DPA (Docosapentaenoic acid) and LA are more than 0.25 , among which DHA was strongly correlated with PC1 $(p<0.0001$, rho $=0.902$ ). PC1 positively and significantly explained TJC count independent of sex and age.

\begin{tabular}{llllll}
\multicolumn{5}{c}{ Table. Parameter } & Estimates by Standard Least Squares \\
& Lower $95 \%$ & Upper $95 \%$ & Std. Beta & p value \\
\hline Intercept & -39.2 & -6.63 & 0 & 0.008 \\
19,20-diHDPA & 4.54 & 16.55 & 0.707 & 0.003 \\
14,15-diHDPE & -26.4 & -6.24 & -0.62 & 0.003 \\
Age & 0.08 & 0.56 & 0.455 & 0.013 \\
Sex & & & & 0.475
\end{tabular}

Conclusions: Since 19, 20-diHDPA (metabolized from DHA) and 14,15-diHETE (from EPA, eicosapentaenoic acid) are both generated by cytochrome P450catalyzed epoxidation followed by conversion to the vicinal diols by epoxide hydrolase, such kind of enzymes might be key molecules connecting lipid metabolism and RA. Although a replication study is inevitable, a certain kinds of lipid and lipid mediator profiles may be associated with disease activity, especially analgesic descriptors such as tender joint count.

References:

[1] Giera M, et al. Lipid and lipid mediator profiling of human synovial fluid in rheumatoid arthritis patients by means of LC-MS/MS. Biochim Biophys Acta. 2012, 1821(11):1415-24.

Acknowledgements: None.

Disclosure of Interest: None declared

DOI: 10.1136/annrheumdis-2017-eular.4052

\section{THU0058 S100A11 PROTEIN IS UP-REGULATED IN PATIENTS WITH IDIOPATIC INFLAMMATORY MYOPATHIES AND IS ASSOCIATED WITH DISEASE ACTIVITY}

L. Andres Cerezo ${ }^{1}$, H. Hulejová ${ }^{1}$, B. Šumová ${ }^{1}$, T. Lennerovaa ${ }^{1}$, M. Klein ${ }^{1}$, H.F. Mann ${ }^{1}$, J. Zámečník ${ }^{2}$, K. Pavelka ${ }^{1}$, J. Vencovský ${ }^{1}$, L. Šenolt ${ }^{1}$. ${ }^{1}$ Department of Rheumatology, 1st Faculty of Medicine, Charles University Prague, Institute of Rheumatology; ${ }^{2}$ Department of Pathology and Molecular Medicine, 2nd Faculty of Medicine, Charles University Prague, University Hospital Motol, Prague, Czech Republic

Background: S100A11 (calgizzarin) is a member of the S100 protein family that participates in regulating number of biologic functions and is associated with oncogenesis and inflammation. Recent data suggest involvement of S100A11 in myocardial damage.

Objectives: The aim of our study was to analyze the expression of S100A11 in patients with idiopathic inflammatory myopathies (IIMs) and its potential association with disease activity parameters and IIMs-related clinical features.

Methods: immunohistochemistry in patients with polymyositis/dermatomyositis (PM/DM, $n=5 / 6)$ and control individuals with myasthenia gravis (MG, $n=5$ ). S100A11 in plasma was measured by ELISA (Biovendor) in 112 patients with IIMs (PM, $n=41 ; D M, n=41$; and cancer associated myositis (CAM), $n=30$ ) and in 42 healthy controls $(\mathrm{HC})$. Patients with PM/DM fulfilled Bohan and Peter diagnostic criteria and CAM was defined as cancer occurring within 3 years of the diagnosis of myositis. Clinical disease activity was assessed by myositis disease activity assessment (MYOACT), physician and patient's global activity using visual analogue scales (VAS), manual muscle testing (MMT) and health assessment questionnaire (HAQ). Muscle enzymes CK, LD, ALT, AST and CRP were measured by routine laboratory techniques. Autoantibodies were detected by immunoprecipitation. 